\title{
Impacto no cotidiano de mulheres com incontinência urinária
}

\author{
Impact on the daily lives of women with urinary incontinence
}

Impacto en el cotidiano de las mujeres con incontinencia urinaria

\author{
Luciana Rosaํ, Maria Teresa Brasil Zanini², Karina Cardoso Gubis Zimermmam³, Mariana Garcia Ghisi', \\ Camila Martins Policarpo ${ }^{4}$, Valdemira Santina Dagostin ${ }^{5}$, Morgana Borba Salvador $^{6}$
}

\begin{abstract}
RESUMO
O objetivo deste estudo foi conhecer o impacto no cotidiano das mulheres com incontinência urinária (IU) e a percepção desse impacto por elas. A IU é uma patologia que provoca perda involuntária de urina. A presente pesquisa teve cunho qualitativo e descritivo e foi desenvolvida em um centro especializado em Urologia no Sul de Santa Catarina, com 15 mulheres com diagnóstico de IU, comprovado por meio do estudo urodinâmico. Utilizou-se para coletar os dados a entrevista semiestruturada. Os dados foram analisados com a técnica de análise simples de conteúdo. Respeitaram-se em todos os momentos da pesquisa os aspectos éticos da Resolução do Conselho Nacional de Saúde (CNS) n. ${ }^{\circ}$ 466/12. Os resultados vêm ao encontro com os de outros estudos: mulheres incontinentes com qualidade de vida afetada em seu cotidiano nas questões profissionais, domésticas, sexuais, físicas e psicológicas. Constatou-se também o desconhecimento das causas que as levaram a desenvolver a IU, já que o problema foi atribuído a fatores que não têm nenhuma fundamentação científica. Observou-se ainda relação importante entre a IU e os processos psicológicos de tristeza, ansiedade e isolamento social. Em conclusão, o estudo evidenciou a influência da IU nos mais variados aspectos do dia a dia da mulher, requerendo dela uma atitude proativa como protagonista principal para a produção e o resgate de sua saúde.
\end{abstract}

DESCRITORES: Incontinência urinária. Saúde da mulher. Qualidade de vida. Estomaterapia.

\begin{abstract}
The objective of this study was to know the impact on women's daily lives with urinary incontinence $(\mathrm{UI})$ and the perception of this impact according to them. The UI is a condition that provokes involuntary loss of urine. This research had qualitative and descriptive nature and was developed in a Urology center in Southern Santa Catarina, in Brazil, with 15 women diagnosed with UI, which was confirmed through the urodynamic study. Semi-structured interview was used to collect the data. Data were analyzed with the simple content analysis technique. We respected in all moments of the research the ethical aspects described on the National Council of Health (CNS) Resolution no. 466/12. The results are in agreement with the ones reached by other studies: incontinent women with affected quality of life in their daily lives in professional, domestic, sexual, physical and psychological issues. Ignorance of the UI causes was also observed, since the problem was attributed to factors that have no scientific basis. It was also seen an important relation between the UI and psychological processes of sadness, anxiety and social isolation. In conclusion, the study showed the influence of UI in various aspects of women's everyday life, requiring their proactive attitude as the main protagonist for the production and recovery of their health.
\end{abstract}

DESCRIPTORS: Urinary incontinence. Women's health. Quality of life. Stomatherapy.

\footnotetext{
1Programa de Pós-Graduação em Ciências da Saúde da Universidade do Extremo Sul Catarinense (Unesc) - Criciúma (SC), Brasil; Secretaria de Estado da Saúde de Santa Catarina - Florianópolis (SC). Brasil; e Centro de Tratamento de Cálculos Renais - Criciúma (SC), Brasil.

Endereço para correspondência: Rua José do Patrocínio, 170, ap. 502 - Centro - CEP: 88801-680 - Criciúma (SC), Brasil. E-mail: bridalu@yahoo.com.br ${ }^{2} U$ nesc - Criciúma (SC), Brasil; e Secretaria de Estado da Saúde de Santa Catarina - Florianópolis (SC), Brasil.

${ }^{3}$ Mestrado na concentração em Filosofia e Enfermagem pela Universidade Federal de Santa Catarina (UFSC) - Florianópolis (SC), Brasil. Curso de

Especialização em Estomaterapia - Criciúma (SC), Brasil.

${ }^{4}$ Curso de Enfermagem da Unesc - Criciúma (SC), Brasil.

${ }^{5}$ Programa de Pós-Graduação em Ciências da Saúde da Unesc - Criciúma (SC), Brasil.

${ }^{6}$ Curso de Enfermagem da Escola Superior de Criciúma (Esucri) - Criciúma (SC), Brasil.

Artigo recebido em: 07/07/2015 - Aceito para publicação em: 23/07/2016.
} 


\section{RESUMEN}

El objetivo de este estudio fue conocer el impacto en la vida cotidiana de las mujeres con incontinencia urinaria (IU) y la percepción de este impacto para ellas. La IU es una condición que resulta en la pérdida involuntaria de orina. Esta investigación tuvo un carácter cualitativo y descriptivo y se desarrolló en un centro especializado en Urología en el Sur de Santa Catarina, Brasil, con 15 mujeres con diagnóstico de IU que fue probada por el estudio urodinámico. La recolección de datos utilizó la entrevista semiestructurada. Los datos fueron analizados con la técnica del análisis de contenido simple. Se respetan en todos los momentos del estudio los aspectos éticos de investigación de la Resolución 466/12 del Consejo Nacional de Salud. Los resultados están de acuerdo con otros estudios: mujeres incontinentes con la calidad de la vida afectada en su vida cotidiana en la ocupación de las cuestiones profesionales, domésticas, sexuales, físicas y psicológicas. Se constató también la ignorancia de las causas que las llevó a desarrollar la IU, como se atribuyeron a factores que no tienen base científica. Todavía había una importante relación entre la IU y los procesos psicológicos de tristeza, ansiedad y aislamiento social. En conclusión, el estudio muestra la influencia de la IU en varios aspectos de la vida cotidiana de las mujeres, lo que requiere su actitud proactiva como la protagonista principal de la producción y la recuperación de su salud.

DESCRIPTORES: Incontinencia urinaria. Salud de la mujer. Calidad de vida. Estomaterapia.

\section{INTRODUÇÃO}

A Sociedade Internacional de Continência (ICS) definiu incontinência urinária (IU) como qualquer perda involuntária de urina, valorizando o relato do paciente, podendo ser classificada, de acordo com seus sintomas, achados clínicos e exames adicionais, em: IU de esforço (IUE), hiperatividade detrusora idiopática (HDI) ou IU mista (IUM) ${ }^{1,2}$.

A IUE ocorre quando há perda involuntária de urina durante o esforço, exercícios físicos, ao espirrar ou tossir. Já a HDI é caracterizada pela queixa de perda involuntária de urina acompanhada ou precedida por urgência, e a IUM dá-se quando acontece queixa de perda involuntária de urina associada à urgência e também aos esforços. Dependendo das condições de saúde, do tipo e do estágio da incontinência, o tratamento pode ser cirúrgico, medicamentoso, fisioterápico ou comportamental. Por intermédio dessas intervenções, é possível que a cura seja obtida, os sintomas sejam minimizados ou a mulher aprenda a lidar melhor com o problema ${ }^{3}$.

O funcionamento da bexiga é comandado por um sistema complexo de nervos que se concentra no trígono e envolve estruturas como: sistema nervoso central, sistema nervoso periférico e estruturas do trato urinário. É a interação entre essas estruturas que estabelece o equilíbrio coordenado e harmônico, determinando a continência urinária ${ }^{4}$.

Os distúrbios psicológicos da IU em mulheres causam aflição e incapacidade, o que têm levado à significativa morbidade, por conta de sentimentos de vergonha e do medo de sofrer rejeição, pois a perda inesperada de urina pode ser uma ameaça a sua autoestima 5 .

Em um estudo transversal, foram avaliadas 505 mulheres com IU que frequentaram os serviços de Urologia e Ginecologia de quatro hospitais no centro de Portugal. Delas, 351 (69,5\%) tiveram HDI; 107 (21,2\%), IUE e 47 (9,3\%), IUM. A IUE foi associada a tabagismo, consumo de álcool, prisão de ventre, gravidez, paridade e infecções vaginais; por sua vez, a IUE, à idade acima de 50 anos, à profissão, ao tabagismo e às mulheres que se sentavam por duas horas ou menos por dia. Já a IUM estava relacionada com idade de 50 anos ou menos, tabagismo, mulheres que se sentavam por duas horas ou menos por dia e aquelas que normalmente transportavam mais de $3 \mathrm{~kg}$ de peso. Impacto negativo na qualidade de vida foi relatado por $501(99,2 \%)$ mulheres. Comparadas com as mais jovens participantes, mulheres com mais de 50 anos apresentaram distúrbios do sono, energia e limitações de desempenho ${ }^{6}$.

A etiologia da IU é multifatorial e a mais comum é a IUE. IU consiste num problema interdisciplinar que se encontra na esfera de interesses de diferentes especialidades. O diagnóstico inicial deve começar por reunir a história médica e pode ser preenchido pelo questionário de qualidade de vida. Atualmente, um dos métodos de diagnóstico não invasivo é o estudo de ultrassom. Numa situação em que o diagnóstico não pode ser colocado ou quando o tratamento cirúrgico se faz necessário, a paciente precisa realizar o estudo urodinâmico ${ }^{7}$.

No Brasil, as pesquisas são poucas e ficam restritas a alguns grupos populacionais, dificultando saber a real 
situação das mulheres brasileiras. Existem na literatura certos métodos de tratamento para a patologia, tanto invasivos quanto não invasivos e cirúrgicos, tais como: cirurgia de Sling, eletroestimulação do assoalho pélvico, terapia com cones vaginais, exercícios perineais e tratamento farmacológico. Os tratamentos são os mesmos de antigamente; o que mudou foram as técnicas cirúrgicas e os equipamentos, que ficaram mais modernos, favorecendo as oportunidades de tratar e melhorar a IU. A enfermagem tem papel de extrema importância no diagnóstico e no tratamento da referida questão. Ela tem os objetivos de incentivar e encorajar as mulheres com IU no tratamento, pois os resultados são alcançados em longo prazo ${ }^{8}$.

A relevância do trabalho aponta para mulheres portadoras de IU que convivem no seu cotidiano com o constrangimento de procurar orientação dos profissionais da saúde, porém somente com comunicação clara é possível identificar-se o problema. Nesse contexto, cabe ao enfermeiro atuar de forma sistematizada e humanizada, instituindo um elo mais forte na busca de intervenções para minimizar o problema. Para tanto, teve-se como propósito conhecer o impacto no cotidiano das mulheres com IU e a sua percepção por elas.

\section{MÉTODOS}

Trata-se de uma pesquisa qualitativa do tipo descritiva, exploratória e de campo. A pesquisa descritiva tem como finalidade "desenvolver, esclarecer, modificar e aprimorar ideias", relatando as características de determinados fenômenos. São incluídas no grupo de pesquisas descritivas as ideias que objetivam levantar "as opiniões, atitudes e crenças de uma população".

O estudo foi realizado em um centro especializado em Urologia, localizado em uma cidade do Sul de Santa Catarina e incluiu 15 mulheres com IU, com diagnóstico confirmado pelo estudo urodinâmico. Tais pacientes foram selecionadas de forma intencional, por intermédio de entrevista semiestruturada, a qual foi transcrita. Adotaram-se como critérios de inclusão: mulheres portadoras de IU, idade superior a 18 anos, não ter déficit cognitivo, aceitar participar de pesquisa e assinar o termo de consentimento livre e esclarecido, seguindo as exigências formais contidas na Resolução no 466/12 do Conselho Nacional de Saúde (CNS).
A pesquisa foi aprovada pelo Comitê de Ética da instituição de ensino Universidade do Extremo Sul Catarinense (Unesc), recebendo autorização sob o número de parecer CAAE-42378915.4.0000.0119.000.

A interpretação dos resultados deu-se pela análise simples de conteúdo. Os dados foram organizados por temas abordados, e fez-se a leitura exaustiva deles, interpretando-os e fundamentando-os à luz das referências disponíveis sobre cada assunto ${ }^{10}$.

\section{RESULTADOS E DISCUSSÃO}

\section{Conhecimento das causas da incontinência urinária}

Apesar de toda a influência que a IU ocasiona na vida das mulheres, ainda é pequeno o número delas que procura assistência profissional de forma precoce. Provavelmente essa negativa se deve a fatores culturais e familiares e, principalmente, ao medo, à vergonha e à falta de conhecimento, como se observou no presente estudo e no encontro com colaboradores do tema em questão.

Nesse contexto, surge a Enfermagem de maneira sistematizada, para realizar sua assistência à mulher incontinente, de modo a proporcionar a tal sujeito governabilidade no processo de produção de saúde.

Entre as entrevistadas, citou-se o desconhecimento dos motivos que as levaram a desenvolver essa patologia. Constatou-se que certas mulheres apresentam algum conhecimento sobre os fatores que provocaram a IU, reportando-os ao seu estado atual.

Outros fatores que podem estar vinculados com a IU são: mulheres brancas, menopausa, cirurgias ginecológicas, constipação intestinal, doenças crônicas, uso de drogas, consumo de cafeína, tabagismo e exercícios físicos ${ }^{11}$.

A proteção do assoalho pélvico e, por consequência, a diminuição dos casos de IU são frequentemente mencionadas como benefícios oriundos do parto cesáreo eletivo, porém a relação entre gestação e tipo de parto com o surgimento da IU não está completamente esclarecida ${ }^{12}$.

\section{Influência nas atividades físicas domésticas}

As tarefas feitas diariamente pela mulher no lar requerem, na maior parte das vezes, algum tipo de esforço físico e atenção. 
Em sua grande parte, exigem excesso de sobrecarga de peso, citando como exemplos mover os móveis para a higienização da casa, lavar roupas e carregar crianças no colo.

A maioria das entrevistadas relatou que ter IU não atrapalha na realização de suas tarefas de casa, e apenas algumas responderam que sua patologia atrapalha em suas tarefas.

Em um estudo de 2007, observou-se interferência dos sintomas nas atividades da vida diária, física e social, como afazeres domésticos, atividades fora de casa, viagens, vida social e visitas a amigos. A maioria das mulheres relatou não se ver afetada ou ter a vida pouco comprometida em função da incontinência. Isso pode ter se dado pelo fato de grande parcela delas já estar em atendimento e/ou de ter havido melhora dos sintomas ${ }^{13}$.

Em um aspecto geral, as mulheres com IU referem-se a limitações em níveis físicos (praticar esporte, carregar objetos) e alterações nas atividades sociais, ocupacionais e domésticas, influenciando negativamente o estado emocional e a vida sexual ${ }^{14}$.

Diante dos resultados e ao encontro da literatura, pode-se sugerir que a baixa influência da IU nas atividades domésticas se dê por conta de as mulheres estarem em seu próprio ambiente familiar, com acesso a banheiro e a sua higiene pessoal. Dessa forma, cria-se e mantém-se um ambiente íntimo em que elas mesmas são protagonistas de seus próprios atos diários.

Exercícios físicos para mulheres portadoras de IU, na maioria das vezes, tornam-se um momento de constrangimento e estresse, pois o esforço físico dispensado acaba acarretando perda urinária. Sabe-se que a maioria das mulheres busca por meio do exercício físico uma maneira de modelar o seu corpo e de melhorar a sua qualidade de vida; no entanto, muitas vezes, os programas de exercícios acabam sendo abandonados em decorrência desse distúrbio durante tais atividades.

A manutenção da prática regular de exercícios físicos e/ou de esportes apresenta fatores protetores sobre a saúde mental e emocional da mulher ao longo e depois da gravidez ${ }^{15}$.

A IU, além de prejuízos ligados à prática de atividades físicas, afeta também, de forma negativa, a qualidade de vida de mulheres que a possuem. Essa patologia pode levar a sentimentos de depressão, ansiedade, diminuição do humor, síndrome do pânico, medo e vergonha. É muito comum que mulheres incontinentes se isolem da sociedade $\mathrm{e}$ do convívio de seus familiares e amigos para evitar situações constrangedoras diante de um episódio de perda de urina ${ }^{16}$.
Sendo assim, a mulher precisa de segurança e conforto na prática diária de exercícios físicos, porém torna-se necessária a adequação para seu estado atual de saúde perante a IU, e o enfermeiro possui conhecimento para abordar esse planejamento de atividades, bem como buscar subsídios efetivos para realizar sua assistência.

\section{Satisfação sexual e repercussões psicológicas}

Quando questionadas se a IU atrapalhava o desempenho de sua vida sexual, apenas uma das entrevistadas disse que sim; as demais negaram que esse problema as prejudicava. Acredita-se que talvez por vergonha ou timidez, ou até por conta da exposição de sua imagem corporal, elas tenham preferido ocultar essa questão, ou mesmo de fato não sintam desconforto para a atividade.

A vida sexual das mulheres está diretamente ligada a sua satisfação, pois qualquer disfunção nesse sentido acaba acarretando problemas de ordem pessoal, familiar, social e psicológica. A IU provoca sentimentos de baixa autoestima, além de interferir na vida sexual e de restringir o contato social e as tarefas domésticas e de trabalho ${ }^{17}$.

A mulher incontinente, sobretudo a casada ou aquela com vida sexual ativa, sofre grande impacto na sua qualidade de vida, porque está sempre com medo ou receio de perder urina em certas situações, como durante a relação sexual, quando sente receio de interromper o ato para urinar e, assim, desagradar o parceiro. No lazer, fica a todo o momento preocupada em localizar um banheiro, tem cuidados excessivos com a higiene íntima, por medo de alguém sentir odor de urina. A qualidade do sono fica comprometida, afinal a mulher se levanta diversas vezes para urinar, o que provoca cansaço e gasto de energia, causando prejuízo no desempenho profissional e outros problemas na vida cotidiana ${ }^{18}$.

Logo, a perda urinária pode ter efeito dramático sobre a qualidade da vida sexual da mulher e levar ao completo abandono da atividade sexual progressivamente, à medida que a patologia evolui.

As repercussões psicológicas da IU em mulheres causam aflição e incapacidades, as quais têm levado à significativa morbidade, em função dos sentimentos de vergonha e medo de sofrer rejeição, pois as mulheres acometidas acabam não procurando ajuda profissional, por ocultamento de sua situação de saúde e por sentimento de perda sentimental, muitas vezes de seu próprio cônjuge 5 . 
A pesquisa reportou uma relação importante entre a IU e os processos psicológicos de tristeza, ansiedade e isolamento social. Percebeu-se que as mulheres se sentem diferenciadas das outras pessoas, vivendo em um mundo totalmente isolado do contexto geral. Por medo ou vergonha, procuram esconder o problema, muitas vezes pelo isolamento social e de isolamento de vínculos afetivos.

A coexistência de sintomas depressivos e IU reflete a influência negativa da referida patologia na qualidade de vida das pacientes que, quando depressivas e incontinentes, apresentam maior probabilidade de gravidade do quadro psiquiátrico ${ }^{18}$. O simples fato de ter vida social implica uma gama de fatores que elas devem considerar para esconder o problema da própria sociedade, trazendo dessa maneira sentimentos avessos a proporcionar a qualidade de vida almejada. Sugere-se que os profissionais enfermeiros voltem seu olhar com evidência à atenção primária, para precocemente identificar e planejar sua assistência de forma sistematizada.

\section{Desempenho profissional}

Observou-se que apenas as mulheres aposentadas não demonstraram muita preocupação em relação às suas atividades, pois estão em seu ambiente domiciliar e com livre acesso ao banheiro e à preservação de sua privacidade, porém aquelas com atividade profissional ativa demonstraram muita preocupação na convivência com a incontinência e a atividade laboral.

A atividade profissional diária requer de cada profissional compromisso, postura, assiduidade e também proatividade. Todos esses fatores são condicionantes para a realização pessoal em relação às atividades laborais, sendo esse tipo de enfoque multidimensional e subjetivo.

Quando esses fatores estão interligados à saúde, podem acarretar ao indivíduo diversas manifestações que venham a influenciar positiva ou negativamente seu desempenho profissional. No caso das mulheres portadoras de IU, muitas vezes em seu ambiente de trabalho, elas passam pelas mais inusitadas situações de constrangimento, por conta de seu estado de saúde. Passam a se preocupar com a disponibilidade de banheiros, envergonham-se com o odor de urina e sentem-se com frequência sujas, chegando a apresentar lesões cutâneas como dermatite amoniacal e infecções urinárias repetidas, afetando diretamente suas atividades ao longo do dia ${ }^{18}$.
Nota-se que o desempenho profissional está muito ligado à condição de saúde da mulher incontinente, item observado principalmente pelas idas consecutivas ao banheiro, interrompendo sobremaneira o rendimento diário de suas atividades laborais, o que sugere que boas condições de saúde interferem de maneira positiva na produção laboral. Mulheres incontinentes necessitam não apenas de um banheiro à disposição, mas acima de tudo de suporte psicológico profissional em seu ambiente de trabalho, que busque soluções efetivas a sua produção de saúde no seu cotidiano.

Os resultados desta pesquisa corroboraram outros trabalhos encontrados na literatura, e constatou-se que a permanência na vida ativa parece estar determinada fortemente pela capacidade física. Essa ideia é reforçada pela forte associação do trabalho a melhores indicadores de autonomia: indivíduos mais saudáveis têm mais chance de ingressar na força de trabalho e também de permanecer no trabalho ${ }^{19}$.

Em relação às mulheres aposentadas, também se torna importante evidenciar que precisam de um olhar voltado para sua condição, pois o fato de estarem em suas residências não as isenta de passar por fortes emoções íntimas e silenciosas em suas atividades do lar em decorrência da perda urinária.

\section{Contenção da perda urinária}

Em relação ao controle de líquidos, a maior parte das entrevistadas não sente necessidade de controlar a ingesta hídrica, mas verificou-se na sua minoria preocupação desse controle, como também a utilização diária de absorvente higiênico. Sabe-se que, com o advento do protetor íntimo, a vida da mulher se tornou muito mais proativa, pois trouxe mais segurança no período menstrual. Porém, seu uso estendeu-se também para as portadoras de IU.

De acordo com um estudo desenvolvido em 2006, observou-se que as mulheres utilizavam alguma estratégia para minimizar a IU: esvaziavam a bexiga em intervalos programados, limitavam a ingestão de líquidos, usavam perfumes ou desodorantes e absorventes para minimizar o odor de urina ou trocavam de roupa ${ }^{11}$.

Recorrer a recursos para a contenção da perda urinária é uma prática comum adotada pelas mulheres nessa condição. $\mathrm{O}$ absorvente e a toalhinha de pano são os métodos mais empregados como recurso de contenção, sendo trocados numa frequência de duas ou mais vezes por $\mathrm{dia}^{20}$.

Diante dos resultados obtidos, considera-se que os absorventes colaboram de certa forma para o controle higiênico 
na IU, contudo não descartam o total desconforto físico da mulher, nem evitam a formação de odores desagradáveis e a manutenção da integridade da pele na região perineal. Para mulheres independentes e com bom estado cognitivo, o absorvente serve como um excelente instrumento de auxílio na IU, no entanto não podemos deixar de citar as mulheres com idade mais avançada, em que a mobilidade física impede a independência da realização de sua higiene corporal.

\section{Tratamentos disponíveis}

A maioria das mulheres entrevistadas não possui conhecimento em relação aos tipos de tratamentos disponibilizados, razão pela qual não procura ajuda profissional, pois acaba imaginando que para tal distúrbio não existe terapia específica. Os tipos de tratamento foram poucos citados, entre eles a perineoplastia. Apenas uma paciente tinha conhecimento sobre a cirurgia de Sling, também indicada para o tratamento das recidivas pós-operatórias da técnica de Burch ${ }^{11}$.

As pacientes incontinentes necessitam receber informações pertinentes para a apropriação do conhecimento e, consequentemente, participar da escolha ideal do tratamento, que pode ser cirúrgico, medicamentoso ou fisioterapêutico ${ }^{1}$.

Observou-se nos resultados que as orientações acerca da IU estão ausentes na maioria das pacientes. Ou seja, a deficiência de informações ainda faz parte do mundo vivenciado pelas mulheres incontinentes. Pergunta-se: quais atores devem estar envolvidos nesse contexto? Apenas o médico assistente? $\mathrm{O}$ ginecologista ou o urologista? O enfermeiro? Qual é o papel da atenção básica para essas pacientes? O direito do conhecimento é de cada usuário do Sistema Único de Saúde (SUS), e cada profissional envolvido precisa ter sua parcela de contribuição. Logo, torna-se necessário o empenho de lideranças do Governo e de gestores de saúde na criação de mecanismos que minimizem o aparecimento, bem como o agravamento, de doenças e distúrbios como a IU.

O SUS necessita, além de uma gestão responsável e de boa vontade política, da participação efetiva dos usuários nas discussões e decisões voltadas para o encaminhamento resolutivo das ações em saúde. Significa dizer, em outras palavras, que os cuidados com a saúde precisam de planejamento, participação e avaliação contínua para melhores resultados e indicadores. É mister entender que os cuidados em saúde são muito mais efetivos quando contemplam práticas integradas e integradoras aos saberes dos diferentes atores sociais ${ }^{21}$.

\section{CONSIDERAÇÕES FINAIS}

Ao abordar o cotidiano das mulheres com IU, pretendeu-se neste trabalho contribuir também de forma dinâmica com novas perspectivas no tratamento e, consequentemente, melhora na qualidade de vida dessas mulheres.

Considerando os resultados obtidos, pôde-se perceber que é possível despertar nas mulheres incontinentes atitudes de autonomia no seu contexto biopsicossocial, por meio principalmente de tomadas de consciência e mudanças de comportamento.

Sendo assim, sugere-se que o enfrentamento de busca da melhoria da qualidade de vida seja um diferencial na produção de saúde, tendo como protagonistas as mulheres que almejam sair do anonimato chamado IU para o palco de atitudes inovadoras no seu dia a dia.

O planejamento da assistência de Enfermagem produz cuidados específicos para que mulheres incontinentes consigam realizar seu autocuidado sem os medos e as frustrações que esse distúrbio possa vir a causar. Nesse ínterim, cabe ressaltar que cada profissional de saúde tem sua parcela de contribuição nesse tipo de cuidado, evidenciando a importância do enfermeiro generalista e especialista em Estomaterapia e Urologia como ator primordial nesse contexto.

O cenário da unidade de saúde, que está tão próxima da população, e o enfermeiro e os demais membros da equipe deveriam oportunizar educação em saúde, no momento em que a mulher incontinente procura a unidade para a busca do entendimento de seu problema e para a sua resolução.

É recomendável que novos estudos sejam construídos no tema abordado para dar continuidade a melhores subsídios que venham a auxiliar de forma significativa a melhoria da qualidade de vida de mulheres incontinentes, influenciando de modo positivo seu cotidiano. Sugerem-se trabalhos de abordagem tanto qualitativa quanto quantitativa, abrangendo um maior número de participantes, bem como um tempo mais longo o de observação sistematizada. 


\section{REFERÊNCIAS}

1. Leroy LS, Lopes MH, Shimo AK. A incontinência urinária em mulheres e os aspectos raciais: uma revisão de literatura. Texto Contexto Enferm. 2012;21(3):692-701.

2. Toledo DD, Dedicação AC, Saldanha ME, Haddad M, Driusso P. Physical therapy treatment in incontinent women provided by a public health service. Fisioter Mov (Impr). 2011;24(2):327-35.

3. Caldas CP, Conceição IR, José RM, Silva BM. Terapia comportamental para incontinência urinária da mulher idosa: uma ação do enfermeiro. Texto Contexto Enferm. 2010;19(4):783-88.

4. Oliveira KA, Rodrigues AB, Paula AB. Técnicas fisioterapêuticas no tratamento e prevenção da incontinência urinária de esforço na mulher. Rev Eletrônica F@pciência. 2007;1 (1):31-40.

5. Higa R, Rivorêdo CR, Campos LK, Lopes MH, Turato ER. Vivências de mulheres brasileiras com incontinência urinária. Texto Contexto Enferm. 2010;19(4):627-35.

6. Jerez-Roig J, Santos MM, Souza DL, Amaral FL, Lima KC. Prevalence of urinary incontinence and associated factors in nursing home residents. Neurourol Urodyn. 2014;35(1):102-7.

7. Wiśniewska B, Marciniak A, Rutkowska-Nawrocka J, Ciećwież M, Szydłowska I, Starczewski A. Urinary stress incontinence: one of basic diseases of modern society. Pol Merkur Lekarski. 2015;38(223):51-4.

8. Oliveira AM, Camargo AC, Schell NC, Navarro EC. Assistência de enfermagem na incontinência urinária na mulher. Rev Múltiplo Saber. 2012.

9. Minayo MC. Pesquisa social: teoria, método e criatividade. 21. ed. Petrópolis: Vozes; 2009.

10. Minayo MC. O desafio do conhecimento: pesquisa qualitativa em saúde. 11. ed. São Paulo: Hucitec; 2008.

11. Higa R, Lopes MH, Turato ER. Psychocultural meanings of urinary incontinence in women: a review. Rev Latino-Am Enferm. 2008;16(4):779-86.
12. Dellú MC, Zácaro PM, Schmitt AC. Prevalência de sintomas urinários e fatores obstétricos associados em mulheres adultas. Rev Bras Fisioter. 2008;12(6):482-87.

13. Oliveira JM, Salgado LB, Schmitt AC, Rosa LC. Correlação entre sintomas urinários e qualidade de vida em mulheres com incontinência urinária. Fisioter Pesq. 2007;14(3):12-7.

14. Rett MT, Simões JA, Herrmann V, Gurgel MS, Morais SS. Qualidade de vida em mulheres após tratamento da incontinência urinária de esforço com fisioterapia. Rev Bras Ginecol Obstet. 2007;29(3):134-40.

15. Lima FR, Oliveira N. Gravidez e exercício. Rev Bras Reumatol. 2005;45(3):188-90.

16. Caetano AS, Tavares MC, Lopes MH, Poloni RL. Influência da atividade física na qualidade de vida e auto-imagem de mulheres incontinentes. Rev Bras Med Esporte. 2009;15(2):93-7.

17. Honorio, MO, Santos SM. Incontinência urinária e envelhecimento: impacto no cotidiano e na qualidade de vida. Rev Bras Enferm. 2009;62(1):51-6.

18. Auge AP, Zucchi CM, Costa FM, Nunes K, Cunha LP, Silva $P V$, et al. Comparações entre os índices de qualidade de vida em mulheres com incontinência urinária submetidas ou não ao tratamento cirúrgico. Rev Bras Ginecol Obstet. 2006;28(6):352-7.

19. Giatti L, Barreto SM. Saúde, trabalho e envelhecimento no Brasil. Cad Saúde Pública. 2003;19(3):759-71.

20. Borba AM, Lelis MA, Brêtas AC. Significado de ter incontinência urinária e ser incontinente na visão das mulheres. Texto Contexto Enferm. 2008;17(3):527-35.

21. Koerich MS, Backes DS, Marchiori MC, Erdmann AL. Pacto em defesa da saúde: divulgando os direitos dos usuários pela pesquisa-ação. Rev Gaúcha Enferm. 2009;30(4): 677-84. 